\title{
Malaria diagnosis in the community: Challenges and potential role of rapid diagnostic tests (RDTs) in the African Region.
}

\author{
Edward M Kamau
}

Family Health International; Box 38835-00623 Nairobi, Kenya. Tel: +254 (20) 2713913/4/5/6; Fax: +254 (20) 2726130;Email:Ekamau@fhi.or.ke/emberu@,hotmail.com

\section{SUMMARY}

At present, malaria rapid diagnostic tests (RDTs) are widely available and used in parts of Asia and Latin America. In Africa, their use has been limited mainly to private health facilities. With the adoption of artemisinin combination therapies (ACTs) by most African countries as first line treatment for malaria, an effective, but expensive treatment is available, and the case for an expanded role for rapid diagnostics in the fight against malaria is clear. Despite this perceived potential role for RDTs, some challenges hinder their introduction and scale-up in the public health sector. Among the requirements are significant investments in policy development, training, infrastructure, and supply chain and quality assurance systems.

[Afr J Health Sci. 2007; 14:114-117]

\section{Introduction}

Malaria is the most important parasitic disease in the world. As many as 1.5 million deaths occur each year, with up to five hundred million clinical episodes, the majority in sub-Saharan Africa [1]. Children under the age of five years and pregnant women bear the major burden of the disease as a result of immature and weakened immunity respectively. Children that survive malaria episodes may suffer from anemia and cerebral complications that affect long-term development [2]. Low birth weight in infants, often a result of malaria infection during pregnancy, undermines their chance of survival [3].

Malaria morbidity, mortality, and transmission can be reduced to insignificant levels if infections can be promptly diagnosed and appropriately treated. Widespread Plasmodium falciparum resistance to common antimalarial drugs in most countries has led to drug policy change to ACTs which are not only more expensive but have complex dosage regimens and probably narrow therapeutic indexes. Since the identification of Plasmodium parasites in human blood in 1880, parasitological diagnosis of malaria has remained and continues to be an essential case management and good clinical practice tool [4]. There are several diagnostic techniques, which include microscopy, RDTs, polymerase chain reaction
(PCR), automated flow cytometry, clinical and presumptive diagnosis [5]. Each of these diagnostic techniques has inherent limitations that have not been fully resolved.

The traditional approach to malaria diagnosis has been by microscope examination of a thick/thin blood smear. This method depends on the availability of a microscope and a trained microscopist. Unfortunately, many malaria-endemic countries of sub-Saharan Africa lack access to both. In addition to laboratory equipment that may be missing or in need of repair, staff training, quality control measures, and supervision, when present, are often inadequate. Malaria RDTs are based on the detection of antigens derived from parasites in lysed patient blood, using a variety of immunochromatographic methods [5].

RDTs for malaria offer a unique opportunity to provide accurate and accessible diagnosis for those suffering in the areas hardest hit by malaria, often those without access to microscopy services. In addition, proper use of quality RDTs has the potential to prevent and manage severe malaria and epidemics [6-8]. These tests do not require elaborate laboratory settings nor highly trained laboratory technicians, both of which are scarce in the countries most affected by malaria. The tests can be performed by a wider range of health providers at all levels of health care with 
minimal training. In addition, the results are available immediately to the health provider. The unit costs for RDTs can be as low as US \$1 [9].

The introduction of rapid malaria diagnostics into malaria control programs is critical in an era of increased multi-drug resistant malaria and strained resources. Rapid diagnostic tests may offer distinct advantages provided that test results are linked to proper treatment choices, the tests kits are used and stored correctly, if they are affordable and quality control and assurance measures are put in place [10].

Health workers can easily learn to perform rapid diagnostic tests and these tests have less waiting-times and indirect costs than microscopy. However, rapid malaria test performance can be limited by issues such as shelf-life and the need for strict manufacturer quality control measures [10].

\section{Rationale for the use of RDTs in the community}

Following the 2000 Abuja Declaration [11], which among other targets required that by 2005 , $60 \%$ of those suffering from malaria, should have prompt access to affordable and appropriate treatment within 24 hours of onset of symptoms, several strategies such as home management of malaria (HMM) have been promoting early recognition and prompt treatment of malaria in the home or community. The recognition of symptoms is based on presumptive diagnosis, which like clinical diagnosis has its own limitations. The era of ACTs availability at home or community will be dawning shortly if not already with us. It is therefore prudent to equip community members with basic tools and knowledge that will complement presumptive diagnosis and contribute to the rational use of ACTs as close to the household as possible. It is anticipated that RDTs may play a significant role in providing a safe and effective parasite-based diagnosis of malaria at community level.

For purposes of this synopsis, "safe and effective use" means the correct application of all critical test steps and appropriate adherence to universal standards for safe blood handling and safe disposal of blood-contaminated wastes. The aim is to highlight some of the challenges that may hinder the utilization of RDTs at community level. Some approaches that would facilitate the introduction and appropriate use of RDTs at the community are suggested.

\section{Challenges}

\section{Technical and operational issues}

Consistent, high accuracy is vital to maintain confidence of health workers and clients in RDT results, and thereby achieve the health benefits and financial savings intended through RDT use. However, it is well documented that accuracy of these tests is heavily dependent upon the user, including preparation of the test and interpretation of results. Studies show up to $30 \%$ errors in preparation and interpretation, and measurable improvement through simple modification of instructions [12-16].

In an attempt to provide an effective alternative to the traditional method of malaria testing, companies worldwide have developed malaria RDTs [9]. While only 2 or 3 RDTs were on the market as recently as 5 years ago, the WHO RDT website now lists more than 20 different manufacturers and over 60 products [9]. Some are intended for diagnosis of $P$. falciparum only, some for $P$. falciparum and $P$. vivax and some for $P$. falciparum and the three other human forms of the plasmodium parasite [5]. However, use in Africa is expected to increase rapidly with The Global Fund To Fight AIDS Tuberculosis and Malaria money becoming available. Most of the tests on the market are cassettes or dipsticks, with cassettes offering superior ease-of-use features.

RDT use by community health workers (CHWs) at the village level presents a tremendous opportunity to improve malaria diagnosis and treatment. However, it also presents a major challenge, since in many areas CHWs have limited training in health, may have limited or no formal education and in some cases may be non-literate. Often, CHWs work in settings where there are limited resources for training and limited or no supervision. Achieving a high level of sensitivity and specificity with RDTs used in this context will depend upon the introduction of a product designed and labeled so that $\mathrm{CHWs}$ can learn to use it safely and accurately without participating in elaborate formal training program and with minimal supervisory reinforcement.

\section{Ethical and regulatory issues}

In most of sub-Saharan countries, CHWs are currently prohibited from performing invasive tests. In such situations, regulatory and ethical approval may be granted for research studies defining the potential role of RDT use at community level and based on the findings, 
appropriate legislation put in place. Although RDTs require less blood than slide microscopy, using an RDT would still expose both the CHW and his/her clients to risk of infection by bloodborne pathogens including HIV/AIDS. For this reason, if CHWs are to use RDTs, they must have adequate information and resources to practice safe blood handling and disposal of blood-contaminated waste, and demonstrate safe use of the tests.

\section{Way Forward}

Since previous research suggests that a brief orientation helps health workers with minimal training and limited literacy to correctly use an RDT job aid, it is crucial that they be developed and tested before substantive policy positions are taken. Job aids can be particularly helpful with activities that involve a pre-defined concrete series of steps to be followed the same way each time the procedure is performed [17]. Use of malaria RDTs and universal precautions for safe blood handling are examples of such procedures. In the Philippines and Laos, volunteer CHWs and villagers using a job aid were able to perform most RDT test steps correctly, but the job aid developed for this latter group of $\mathrm{CHWs}$ was unable to address two key issues: checking for test viability (expiry date and exposure to humidity) and waiting for the recommended time before reading test results [18]. Further, accurate interpretation of test results by $\mathrm{CHWs}$ only reached acceptable levels when the job aid was supplemented with an orientation program on use and interpretation of the test. Further, the use of local language(s) on the job aids enhanced their use and facilitated the orientation program [19].

Based on results from the Philippines and Laos, the use of spot color or full color drawings or black and white or color photographs in RDT job aids has been demonstrated to be an effective pictorial orientation tool.

\section{Conclusions}

While RDTs can be effective in clinical settings when used by trained personnel, they also have great potential in rural areas with limited access to health facilities and health professionals. If RDTs can be used with a high level of sensitivity and specificity by CHWs at the village level, it will be possible to make a positive diagnosis in a much greater proportion of malaria cases. Results will include a shorter lag time between the onset of symptoms and the beginning of treatment, less suffering on the part of the infected individual, and reduced transmission potential. It may also lead to a significant cost savings by avoiding unnecessary use of antimalarials, and will therefore contribute to reduce resistance development.

Finally, it would be important to establish the role and cost-effectiveness of RDTs in special situations such as those caused by conflicts or environmental catastrophes, including the displacement of non-immune populations into malaria-endemic areas among other complex emergencies [20-21].

\section{References}

1. World Health Organization and UNICEF. World Malaria Report 2005. Roll Back Malaria, World Health Organization and UNICEF. http://rbm.who.int/wmr2005

2. Carter JA, Ross AJ, Neville BG, Obiero E, Katana K, Mung'ala-Odera V, Lees Ja and Newton CR. Developmental impairments following severe falciparum malaria in children. Tropical Medicine and International Health. 2005; 10: 3-10.

3. Steketee RW, Nahlen BL, Parise ME and Menendez $\mathrm{C}$. The burden of malaria in pregnancy in malaria-endemic areas. American Journal Tropical Medicine Hygiene. 2001; 64(1-2 Supplement): 28-35.

4. World Health Organization. New Perspectives: Malaria Diagnosis. 2000; WHO/MAL/2000.1091.

5. Anthony Moody. Rapid diagnostic tests for malaria parasites. Clinical Microbiology Reviews. 2002; 15: 66-78.

6. Premji Z, Minjas JN and Schiff CJ. L Laboratory diagnosis of malaria by village health workers using the rapid manual ParaSight-F test. Transactions Royal Society of Tropical Medicine and Hygiene. 1994; 88: 418.

7. Singh N, Valencha M and Sharma VP. Malaria diagnosis by field workers using an immunochromatographic test. Transactions Royal Society of Tropical Medicine and Hygiene. 1997; 91: 396397.

8. Verle P, Bink LN, Lieu TT, Yen PT and Coosemans M. ParaSight ${ }^{\circledR} F$ test to diagnose malaria in hypo-endemic areas 
and epidemic prone regions of Vietnam. Tropical Medicine and International Health. 1996; 1: 794-796.

9. $\quad$ http://www.wpro.who.int/rdt/link8.cfm Accessed 26 March 2007

10. Bell D, Wongsrichanalai $\mathrm{C}$ and Barnwell JW. Ensuring quality and access of malaria diagnosis: how can it be achieved? Nature Reviews, Microbiology. 2006; 4: 682-695.

11. "The Abuja Declaration on Roll Back Malaria in Africa". African Heads of State. Abuja, Nigeria. April $25^{\text {th }} 2000$. [http://www.rbm.who.int/docs/Abuja declaration. PDF].

12. Funk MP, Schlagenhauf P, Tschopp A and Steffen R. MalaQuick versus ParaSight ${ }^{\mathbb{B}} \mathrm{F}$ test as a diagnostic aid in travelers' malaria. Transsctions Royal Society Tropical Medicine Hygiene. 1999; 93: 268-72.

13. Trachsler M, Schlagenhauf $P$ and Steffen R. Feasibility of a rapid dipstick antigen-capture assay for self-testing of travelers' malaria. Tropical Medicine and International Health. 1999; 4:442447.

14. Jelinek T, Grobusch MP, and Nothdurft HD. Use of dipstick tests for the rapid diagnosis of malaria in non-immune travelers. Journal Travel Medicine. 2000; 7:175-179.

15. Whitty CJM, Armstrong $\mathrm{M}$ and Behrens $\mathrm{RH}$, Self-testing for falciparum malaria with antigen-capture cards by travelers with symptoms of malaria. American Journal Tropical Medicine Hygiene. 2000; 63: 295-297.

16. Rennie W, Phetsouvanh R, Lupisan S, Vanisaveth V, Hongvanthong B, Phompida S, Alday P, Fulache M, Lumagui R, Jorgensen P, Bell D and Harvey S. Minimizing human error in malaria rapid diagnosis: clarity of written instructions and health worker performance. Transactions Royal Society of Tropical Medicine and Hygiene. 2007; 101: 9-18.

17. Knebel E, Lundahl S, Raj-Edward A, Abdallah H, Ashton J and Wilson N. The Use of Manual Job Aids by Health Care Providers: What Do We Know? Quality Assurance Project. Operations Research Issue Paper 2000; 1: 1-24. http://www.qaproject.org. Accessed 27 March 2007.
18. Rennie W and Harvey SA. Developing and testing a generic job aid for malaria rapid diagnostic tests (RDTs). Field Report, 2004. Quality Assurance Project in collaboration with WHO/WPRO, Research Institute for Tropical Medicine, Philippines and Ministry of Health, Lao People's Democratic Republic. A WHO/WPRO publication:

http://www.wpro.who.int/rdt/link8.cfm accessed 26 March 2007.

19. Tavrow P, Knebel E and Cogswell L. Using quality design to improve malaria rapid diagnosis test in Malawi. Operations Research Results. 2000; 1 (4). Published for the United States Agency for International Development (USAID) by the Quality Assurance Project (QAP): Bethesda, Maryland, USA. $\quad$ http://www.qaproject.org. Accessed 27 March 2007.

20. Hashizume M, Kondo H, Murakami T, Kodama M, Nakahara S, Lucas ME and Wakai S. Use of rapid diagnostic tests for malaria in an emergency situation after the flood in Mozambique. Public Health. 2006; 120: 444-447.

21. Rolland E, Checchi F, Pinoges L, Balkan S, Guthmann JP and Guerin PJ. Operational response to malaria epidemics: are rapid diagnostic test cost-effective? Tropical Medicine and International Health. 2006; 11:398-408. 